\title{
Antinociceptive and neuroprotective effects of bromelain in chronic constriction injury-induced neuropathic pain in Wistar rats
}

\author{
Ahmed Olalekan Bakare and Bamidele Victor Owoyele \\ Neuroscience and Inflammation Unit, Department of Physiology, Faculty of Basic Medical Sciences, University of Ilorin, Ilorin, Nigeria
}

Received June 7, 2019

Revised September 21, 2019

Accepted September 22, 2019

\section{Correspondence}

Ahmed Olalekan Bakare

Neuroscience and Inflammation Unit,

Department of Physiology, Faculty of

Basic Medical Sciences, University

of Ilorin, Ilorin, Kwara State 240003,

Nigeria

Tel: +234-8039101606

Fax: +234-031-221593

E-mail: wlabwise@gmail.com
Background: The continuous search for a novel neuropathic pain drug with few or no side effects has been a main focus of researchers for decades. This study investigated the antinociceptive and neuroprotective effects of bromelain in sciatic nerve ligation-induced neuropathic pain in Wistar rats.

Methods: Forty-eight Wistar rats randomly divided into eight groups comprised of six animals each were used for this study. Peripheral neuropathy was induced via chronic constriction of the common sciatic nerve. Thermal hyperalgesic and mechanical allodynia were assessed using a hotplate and von Frey filaments, respectively. The functional recovery and structural architecture of the ligated sciatic nerve were evaluated using the sciatic functional index test and a histological examination of the transverse section of the sciatic nerve. The neuroprotective effects of bromelain were investigated in the proximal sciatic nerve tissue after 21 days of treatment.

Results: Bromelain significantly $(P<0.05)$ attenuated both the thermal hyperalgesia and mechanical allodynic indices of neuropathic pain. There were improvements in sciatic function and structural integrity in rats treated with bromelain. These rats showed significant $(P<0.05)$ increases in sciatic nerve nuclear transcription factors (nuclear factor erythroid-derived-2-related factors-1 [NrF-1] and NrF-2), antioxidant enzymes (superoxide dismutase and glutathione), and reduced membranelipid peroxidation compared with the ligated control group.

Conclusions: This study suggest that bromelain mitigated neuropathic pain by enhancing the activities of nuclear transcription factors (NrF-1 and NrF-2) which increases the antioxidant defense system that abolish neuronal stress and structural disorganization.

Key Words: Antioxidants; Bromelain; Hyperalgesia; Neuralgia; Neuroprotection; NF-E2-Related Factor 2; NFI Transcription Factors; Nuclear Respiratory Factor 1; Rats, Wistar

\section{INTRODUCTION}

Neuropathic pain is manifested as a result of damage or lesions in the sensory nervous system. It is characterized by allodynia and hyperalgesia. It is one of the major devastat- ing conditions with long-term impairment of the affected patients' social life and overall quality of life. This class of pain affects $7 \%$ to $9 \%$ of the world population [1]. Recently, the search for a treatment for neuropathic pain with few or no complications has been intensified. Non-steroidal anti- (c) This is an open-access article distributed under the terms of the Creative Commons Attribution Non-Commercial License (http://creativecommons.org/licenses/by-nc/4.0/), which permits unrestricted non-commercial use, distribution, and reproduction in any medium, provided the original work is properly cited.

(c) The Korean Pain Society, 2020
Author contributions: Ahmed Olalekan Bakare: Study conception; Bamidele Victor Owoyele: Supervision. 
inflammatory drugs, opiates, tricyclic antidepressant, and anticonvulsant drugs dominate the preferred choice used in clinical practices for the treatment of neuropathic pain. However, limited efficacy and unacceptable side effects have hindered the acceptability of these categories of drugs [2].

Reactive superoxide molecules have been implicated in the development and maintenance of neuropathic pain. Studies have suggested that endoneural lipid peroxidation (LPO) increases as a consequence of chronic constriction injury (CCI) to the sciatic nerve [3]. The levels of superoxide have been found to be increased in the dorsal horn of the spinal cord and dorsal root ganglia following sciatic nerve constriction [4]. Increased reactive oxygen species (ROS) initiate and maintain both peripheral and central sensitivity which result in hyperalgesic symptoms in neuropathic pain [5]. Systemic injection of a ROS scavenger such as phenyl-N-tert-butylnitrone, 5,5,-dimethyl1-pyrroline-N-oxide, vitamin E, and 4-hydroxy-2,2,6,6,tetramethylpiperidone- $\mathrm{N}$-oxyl have been demonstrated to ameliorate neuropathic pain symptoms [6]. Nuclear factor erythroid-derived-2-related factors-1 (NrF-1) and NrF-2 are regulatory proteins that play active roles in promoting antioxidant enzymes synthesis $[7,8]$. They are tightly regulated cytoplasmic factors that become activated under stressful conditions (oxidative stress). When activated, they translocate into the nucleus where they bind with the antioxidant responding elements of the DNA to modulate the expression of genes involved in oxidative stress [9].

Bromelain, a major protease enzyme found in pineapple (Ananas comosus), has recently gained the attention of researchers for its therapeutic purposes. It is extracted from both the stem and fruit of the pineapple. Traditionally, it has been therapeutically used as an anti-inflammatory agent, as a modulator of tumor growth, for healing of wounds, and for the treatment of arthritis, episiotomy, and muscular pain $[10,11]$.

The antinociceptive effects of bromelain have been well documented in both clinical and animal studies of acute and inflammatory pain [12]. Its effect and mechanism of actions in neuropathic painful conditions have not been reported. Likewise, the role of NrF-1 and NrF-2 in a CCI model of neuropathic pain has not been investigated. Hence, this study assessed the antinociceptive effects of bromelain in sciatic nerve ligation-induced neuropathic pain and its neuroprotective capabilities in the ligated sciatic nerve.

\section{MATERIALS AND METHODS}

\section{Animals}

Male Wistar rats weighing 150 to $180 \mathrm{~g}$, and bred at Central Animal House, Osun State University, Nigeria, were used for the study. The rats were housed in the animal facilities of the Faculty of Basic Medical Sciences, University of Ilorin, Nigeria, under standard conditions of a 12-hour light and dark cycle with free access to food and water. They were acclimatized to the animal facility for 14 days before the commencement of the experiments. All experimental protocols were approved by the University of Ilorin Ethical Review Committee, with Approval Number: UERC/ ASN/2017/936.

\section{CCI}

Peripheral neuropathy was induced by chronic constriction of the major sciatic nerve (CCI) in rats by slightly modifying the method described by Jiang et al. [13]. In brief, male rats were anesthetized with ketamine hydrochloride (100 mg/kg, intraperitoneal injection [i.p.]). The hairs on the lower back and thigh of the rats were shaved. The skin of the lateral surface of the right thighs were incised, and cuts were made bluntly through the biceps. The sciatic nerves were then exposed and freed from adhering tissues. Four ligatures (silk sutures size 4-0) were placed loosely around each sciatic nerve without disrupting the epineural blood supply. After performing the ligation, muscular and skin layers were sutured in layers with catgut and suturing thread respectively. Sham operations were performed similarly to the procedure described above with the exception of placing suture silk around the sciatic nerve. Following this, the rats were allowed to recover with an ad libitum supply of food and water.

\section{Drug and treatment schedule}

Animals were randomly divided into eight groups comprising of six rats each. Bromelain, normal saline, or gabapentin were administered orally to the animals for 21 consecutive days after surgery. Pretreated bromelain groups were administered with bromelain for seven consecutive days prior to the ligation of the sciatic nerve and was followed-up with the same dose for the next 21 consecutive days after sciatic nerve ligation. The summary of the grouping and treatments are as follows:

Group A (control): $10 \mathrm{~mL} / \mathrm{kg}$ of normal saline (per os [p.o.]) Group B (sham control): $10 \mathrm{~mL} / \mathrm{kg}$ of normal saline (p.o.) Group C (ligated control): $10 \mathrm{~mL} / \mathrm{kg}$ of normal saline (p.o.) Group D (ligated reference): $30 \mathrm{mg} / \mathrm{kg}$ of gabapentin 
Group E: Low dose of bromelain (30 mg/kg)

Group F: High dose of bromelain (50 mg $/ \mathrm{kg}$ )

Group G: Pretreated low dose of bromelain ( $30 \mathrm{mg} / \mathrm{kg}$ )

Group H: Pretreated high dose of bromelain $(50 \mathrm{mg} / \mathrm{kg}$ )

Bromelain powder 3,000 gelatin digesting units per gram was obtained from KAN Phytochemicals (Rai, India), PVT Ltd. (Bangalore, India), and Maple Lifesciences (Subhash Nagar, India). Gabapentin was a product of Teva UK limited (Eastbourne, UK). Bromelain and gabapentin were dissolved in normal saline $(0.9 \% \mathrm{NaCl})$ and administered orally. The dosages were carefully selected based on literature reports $[12,14]$.

\section{Behavioral tests}

Behavioral pain tests (thermal hyperalgesia and mechanical allodynia tests) were carried out thirty minutes after administration of either bromelain, gabapentin, or normal saline. The rats were acclimatized to the experimental room and the surface of each piece of equipment used thirty minutes prior to each test to reduce fear and anxiety in the animals. All the behavioral tests were assessed before the sciatic nerve ligation and after the ligation at the 3rd, 7th, 14th, and 21st days after treatment.

\section{Thermal hyperalgesia test}

Thermal hyperalgesia was assessed using a hotplate test (DB-1A; Wincom Company Ltd., Hunan, China) model. The development of thermal hypersensitivity associated with neuropathic pain was measured chronologically using the paw withdrawal latency (PWL) of the rat placed on the surface of the hotplate maintained at $55^{\circ} \mathrm{C} \pm 0.5^{\circ} \mathrm{C}$. The time taken by the rat to lick the ligated hind limb or jump from the surface of the hotplate was taken as PWL. A cutoff time of 20 seconds was imposed on each animal in order to avoid paw tissue injury.

\section{Mechanical allodynia test}

von Frey filaments (Cat No: 37450-275; Ugo Basile, Gemonio, Italy) were used to assess mechanical hypersensitivity by measuring the paw withdrawal threshold to the applied stimuli as described by Zhu et al. [15]. A series of 12 von Frey filaments were used in a sequential order that have approximately equal logarithmic incremental bending forces (equivalent to 1, 1.4, 2, 4, 6, 8, 10, 15, 26, 60, 100, and $180 \mathrm{~g}$ forces, respectively). The rats were situated in a transparent perspex box with a wire mesh floor for at least 15 minutes of habituation time before the behavioral test. The filaments were applied to the plantar surface of each hind paw in ascending order. At each force, the behavior of each rat was observed three times and the allodynic mechanical threshold was defined as the minimal force that caused at least two withdrawals, sudden flinching, or paw licking observed out of three consecutive trials.

\section{Sciatic functional index (SFI) test}

The SFI was assessed to evaluate the progress in the autonomy of animals as described by Zhang et al. [16]. In brief, animals were subjected to walking-track analysis and SFI was estimated from the printed foot image using the formula derived by Bain et al. [17]. The trial was done in an $8.2 \times 42 \mathrm{~cm}$ darkened corridor with a floor covered with white sheet of paper. The rat's hind limb was dipped in black ink and the rat was allowed to walk freely in the corridor. Printed-length factor (PLF), toe-spread factor (TSF), and intermediate toe-spread factor (ITF) from the image of the foot were used in estimating the SFI.

$$
\mathrm{SFI}=-38.3 \times \mathrm{PLF}+109.5 \times \mathrm{TSF}+13.3 \times \mathrm{ITF}-8.8
$$

An SFI of 0 is normal and of -100 indicates total impairment.

\section{Biochemical assay}

On the 22nd day after surgery, the animals were euthanized using an i.p. of ketamine. The ligated sciatic nerve was excised and washed with cold phosphate buffer solution. Tissue homogenates were prepared with ice cold phosphate buffer ( $\mathrm{pH}$ 7.4) and centrifuged. The supernatant of homogenates was employed in estimating total LPO in form of malondialdehyde (MDA), superoxide dismutase (SOD), and reduced glutathione (GSH) level using laboratory kits and a spectrophotometer. Nuclear transcription factors were estimated by the enzyme-linked immunosorbent assay (ELISA) method.

\section{Estimation of LPO}

The assay method of Hunter et al. [18], modified by Gutteridge and Wilkins [19] was adopted. MDA, a product of LPO, was estimated by adding trichloroacetic acid-thiobarbituric acid-hydrochloric acid (TCA-TBA-HCl) reagent to the supernatant from sciatic nerve homogenates. The solution was heated for 15 minutes in a boiling water bath. After cooling, the flocculent precipitate was removed by centrifugation at 1,000 rpm for 10 minutes. The absorbance of the supernatant was measured at $532 \mathrm{~nm}$.

\section{Estimation of SOD}

This was determined in accordance with method used by Misra and Fridovich [20]. Adrenaline auto-oxidizes rapidly 
in an aqueous solution of adrenochrome, whose concentration can be determined at $480 \mathrm{~nm}$ using a spectrophotometer.

\section{Estimation of reduced GSH}

Reduced GSH was measured according to the method of Ellman [21]. An equal quantity of the supernatant from the sciatic nerve homogenate was mixed with $10 \%$ TCA and centrifuged to separate the proteins. The supernatant obtained was mixed with a phosphate buffer (pH 8.4), 5, 5-dithio, bis (2-nitrobenzoic acid) and double-distilled water. This mixture was vortexed and the absorbance was taken at $412 \mathrm{~nm}$ within 15 minutes.

\section{Estimation of nuclear transcription factors}

Homogenate from the sciatic nerves was utilized for the determination of NrF-1 and NrF-2. These transcription factors were determined using their respective ELISA kits (Cayman Chemical, Ann Arbor, MI) and procedure was followed according to the manufacturer instructions.

\section{Histological examination}

The transverse sections of the sciatic nerve were stained with hematoxyline and eosin after 21 days of treatment as described by Muthuraman et al. [22]. Briefly, the proximal sections of the ligated sciatic nerves were excised and fixed with $10 \%$ formalin solution for 2.4 hours. The nerve sections were then blocked and further sectioned transversely into $5 \mu \mathrm{m}$, stained and observed under a high-power light microscope $(\times 400)$.

\section{Statistical evaluation}

All results were expressed as mean \pm standard error of the mean. SPSS ver. 20.0 software (IBM Corp., Armonk, NY) was used to analyze all data. Two-way analysis of variance (ANOVA) was used to analyze all the behavioral tests except the von Frey filament test that was not normally distributed but was transformed. One-way ANOVA was used to analyze all biochemical parameter and the von Frey filament test. Bonferroni post hoc multiple comparisons were used to determine the level of significance. The significant level was set at $P<0.05$.

\section{RESULTS}

\section{Pre-surgical behavioral test}

None of the animals showed any symptoms of thermal hyperalgesia or mechanical allodynia prior to the surgical procedure. There were no significant differences when comparing groups on the mechanical allodynia and thermal hyperalgesia tests except the groups that were pretreated with bromelain as represented in Fig. 1.

\section{Thermal hyperalgesia test}

Bromelain effectively reduced thermal hyperalgesia which was evidenced by increases in the PWL in the hotplate test (Fig. 1). Pretreated rats showed the most efficient thermal anti-nociceptive effects of bromelain, as this group was observed to have the highest latency (PWL) on the hotplate test. In these groups, there was no indication of thermal hyperalgesia when compared with both the ligated control groups (19.80 $\pm 0.59 \mathrm{sec} v s .4 .96 \pm 0.48 \mathrm{sec}, P<0.05)$ and control groups $(19.80 \pm 0.59 \mathrm{sec} v s .9 .13 \pm 0.36 \mathrm{sec}, P<$ $0.05)$. The bromelain administered groups showed a dose dependent response to thermal stimulus. It was noted that the PWL of the sham group increased significantly $(P<$ $0.05)$ compared with the ligated control group on the 7th, 14th and 21st day of post-surgical tests only as indicated in Fig. 1.

\section{Mechanical allodynia test}

CCI resulted in a high degree of mechanical allodynia in ligated animals as indicated in Fig. 2. Mechanical allodynia was highly pronounced in the ligated control group ([3.67 $\pm 0.33 \mathrm{~g}]-[5.67 \pm 0.62 \mathrm{~g}]$ average bending force). It was observed that groups administered with different doses of bromelain and gabapentin were effective in mitigating mechanical allodynia. The highest therapeutic effect of bromelain was observed on the 14th and 21st day postsurgical operation.

\section{SFI test}

The defects in sciatic nerve function observed in ligated animals were significantly lower $(P<0.05)$ compared with the control groups. Bromelain significantly $(P<0.05)$ improved the sciatic nerve function as indicated in Fig. 3. It was observed that high dose bromelain as well as pretreatment with bromelain enhanced the sciatic function progressively. The improvement was significantly higher $(P<$ $0.05)$ than the reference control ( $30 \mathrm{mg} / \mathrm{kg}$ gabapentin) on the 21st day post-surgical SFI test. However, neither the ga- 
A

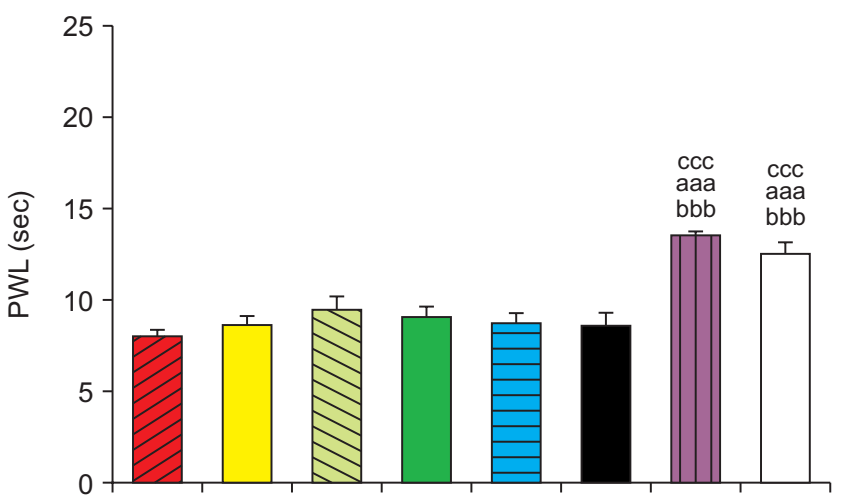

C

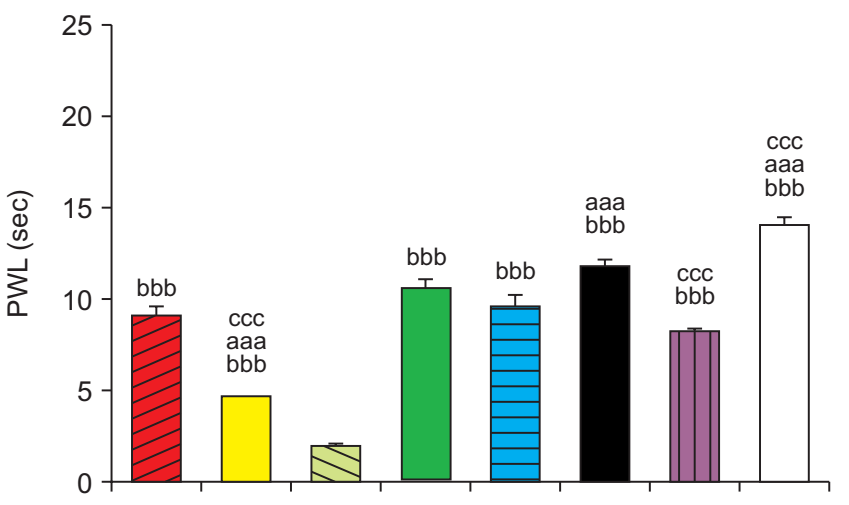

E

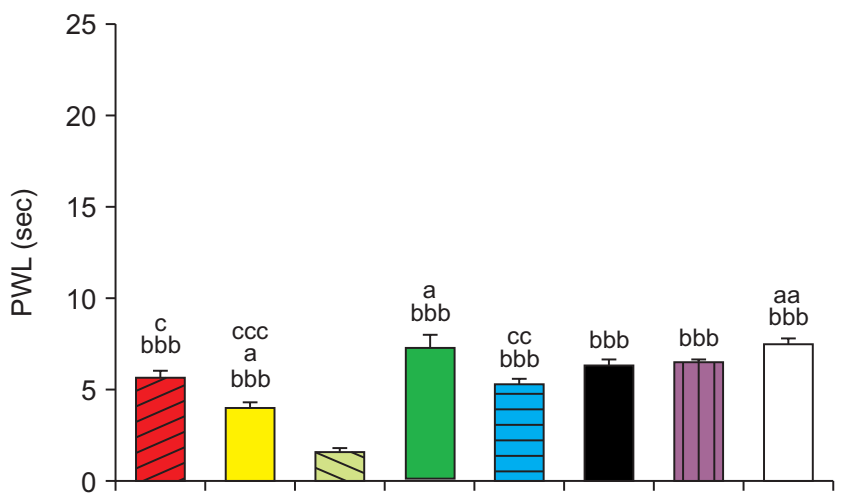

B

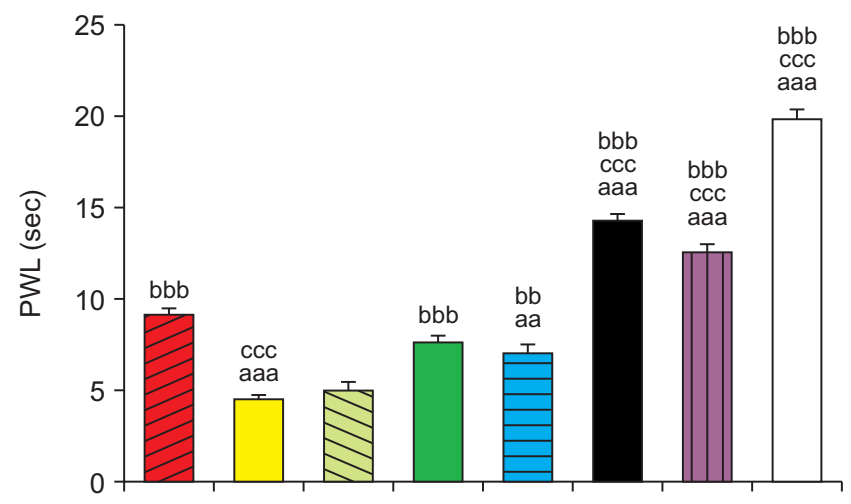

D

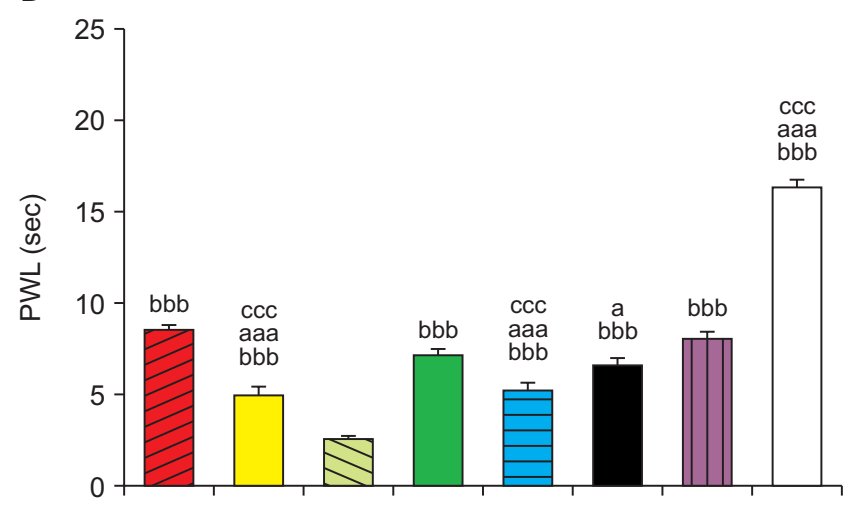

Normal control

Sham control

Ligated control

$30 \mathrm{mg} / \mathrm{kg}$ gabapentin

$30 \mathrm{mg} / \mathrm{kg}$ bromelain

$50 \mathrm{mg} / \mathrm{kg}$ bromelain

Pretreated $30 \mathrm{mg} / \mathrm{kg}$ bromelain

Pretreated $50 \mathrm{mg} / \mathrm{kg}$ bromelain

Fig. 1. Bromelain reversed thermal hyperalgesia in sciatic nerve-induced neuropathic pain. (A) Presurgical hotplate test. (B) Third day post-surgery hotplate test. (C) Seventh day post-surgery hotplate test. (D) Fourteenth day post-surgery hotplate test. (E) Twenty first day post-surgery hotplate test. Each value represents the mean \pm standard error of the mean of each group. PWL: paw withdrawal latency. ${ }^{\mathrm{a}} P<0.05,{ }^{\text {aa }} P<0.01$, ${ }^{\text {aaa }} P<0.001$ compared with

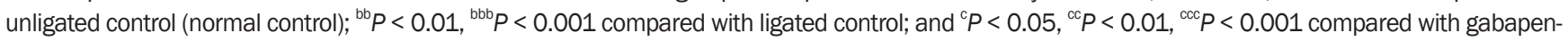
tin (reference control).

bapentin nor the bromelain administered group returned to the basal level on the SFI within 21 days of treatment.

\section{Effect of bromelain on antioxidant enzymes (SOD, GSH) and MDA level in CCI rats}

The activities of GSH and SOD increased significantly $(P<0.05)$ in the homogenate of the ligated sciatic nerves of the groups administered with bromelain and gabapentin compared with the ligated control group as indicated in
Fig. 4A, B. The GSH level was decreased significantly $(P<$ $0.05)$ in the ligated group compared with all other groups. The effect of bromelain on concentration level of GSH was more pronounced compared with the gabapentin administered group as indicated in Fig. 4A. Likewise, there was a significant $(P<0.05)$ increase in the SOD activities of the groups administered with bromelain compared with the control group $(18.68 \pm 0.45 \mathrm{U} / \mathrm{mg}$ protein vs. $6.58 \pm 0.18 \mathrm{U} /$ mg proteins). Bromelain significantly $(P<0.05)$ reduced LPO level compared with the ligated control group. There 

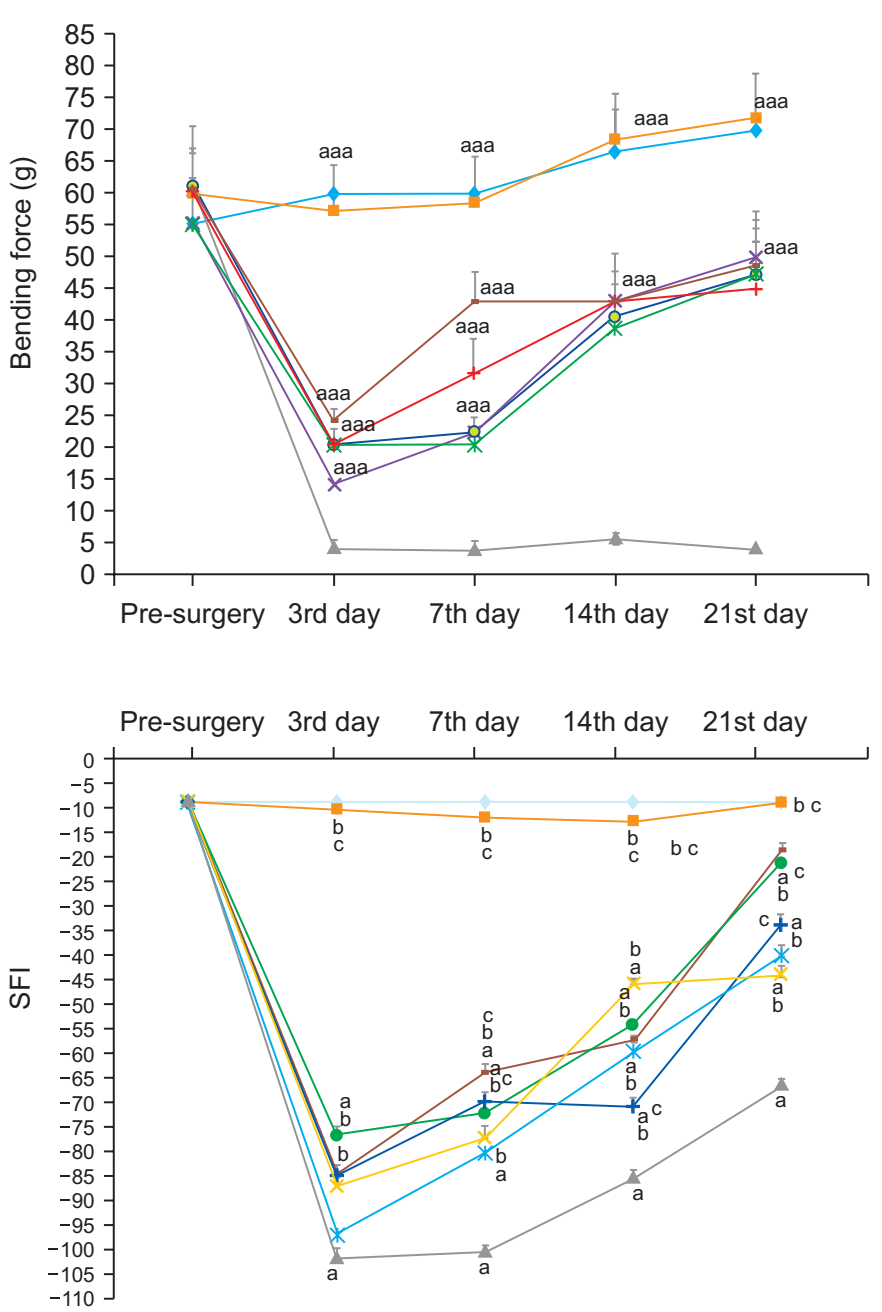

was a high level of MDA in the sciatic homogenate of the ligated control group $(3.06 \pm 0.05 \mathrm{~nm} / \mathrm{mg})$ which was significantly higher $(P<0.05)$ compared with all other groups.

\section{Effect of nuclear transcription factors}

CCI led to a reduction in the activities of nuclear transcription factors (NrF-1 and NrF-2) in the sciatic nerve (Fig. 5). At the end of 21 days of bromelain administration, there were significant $(P<0.05)$ increases in NrF-1 $(14.42 \pm 0.32$ $\mu \mathrm{g} / \mathrm{mL} / \mathrm{mg}$ protein vs. $11.05 \pm 0.24 \mu \mathrm{g} / \mathrm{mL} / \mathrm{mg}$ protein) and $\mathrm{NrF}-2(34.50 \pm 1.61 \mu \mathrm{g} / \mathrm{mL} / \mathrm{mg}$ protein vs. $18.26 \pm 1.06 \mu \mathrm{g} /$ $\mathrm{mL} / \mathrm{mg}$ protein) compared with the ligated control group. Bromelain totally reversed the effect of CCI on NrF-land NrF-2 activities back to the basal level. These effects were more pronounced in groups administered with a high dose of bromelain. There was no significant difference between the pretreated and post-treated bromelain groups.

\section{Histological assessment}

CCI induced axonal degeneration of the sciatic nerve

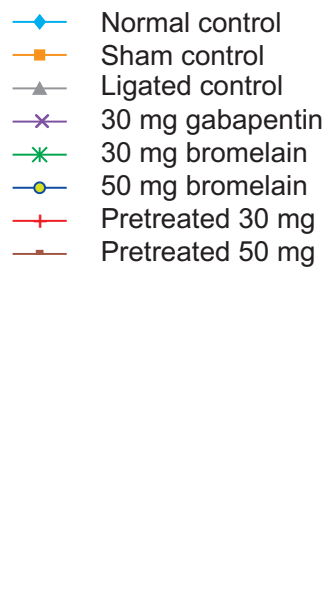

Fig. 2. Bromelain reversed mechanical allodynia in sciatic nerve-induced neuropathic pain. ${ }^{\text {aaa }} P<0.001$ compared with ligated control.

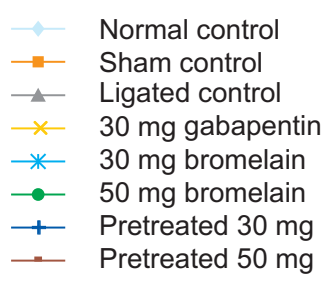

Fig. 3. Bromelain improved the SFI in sciatic nerve-ligated hind limb. SFI: sciatic functional index. ${ }^{\mathrm{a}} P<0.05$ compared with unligated control (normal control); ${ }^{\mathrm{b}} \mathrm{P}<$ 0.05 compared with ligated control; and ${ }^{\mathrm{c}} P<0.05$ compared with gabapentin (reference control).
(Fig. 6C). This was manifested by an increased occurrence of swollen, proliferated myelinated and non-myelinated neurons (Fig. 6C). There was a significant reduction in the number of Schwann's cells observed compared with the normal control group. Bromelain administration mitigated these observed features (Fig. 6E, F). There were increases in the number of myelinated neurons and the numbers of swollen myelinated and non-myelinated neurons were grossly abated in the group treated with bromelain. The sciatic nerve of the pretreated bromelain group (Fig. 6G, H) showed nearly fully recovered structural integrity similar to the unligated control group.

\section{DISCUSSION}

The current study investigated the antinociceptive effect and possible mechanism of action of orally administered bromelain in sciatic nerve ligated neuropathic pain in Wistar rats. The anti-nociceptive effect of bromelain was investigated using tactile and thermal stimuli. Mechanical allodynia and thermal hyperalgesia are familiar symp- 
A

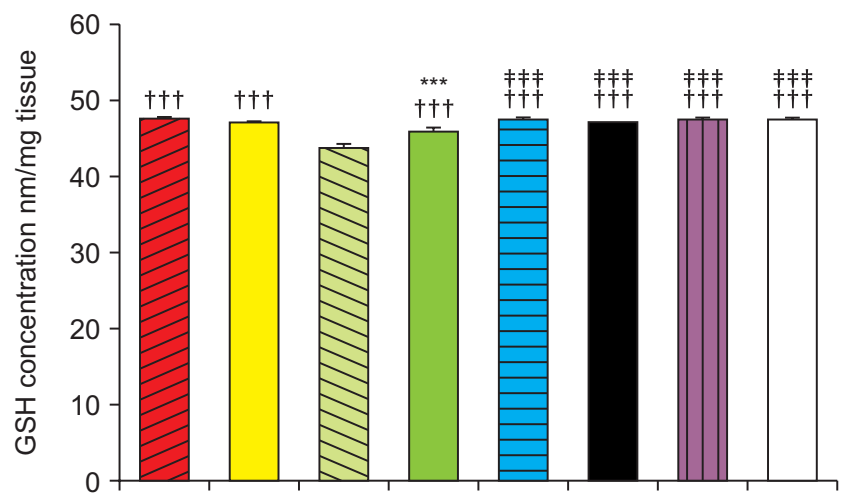

C

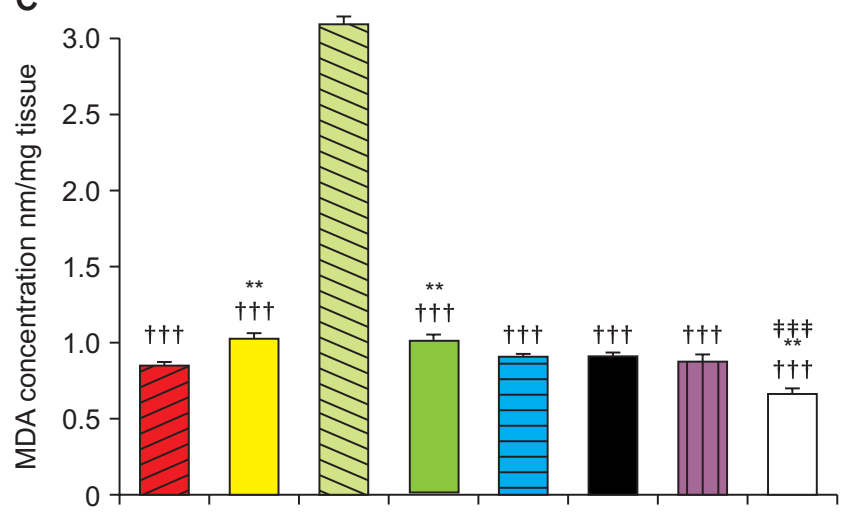

B

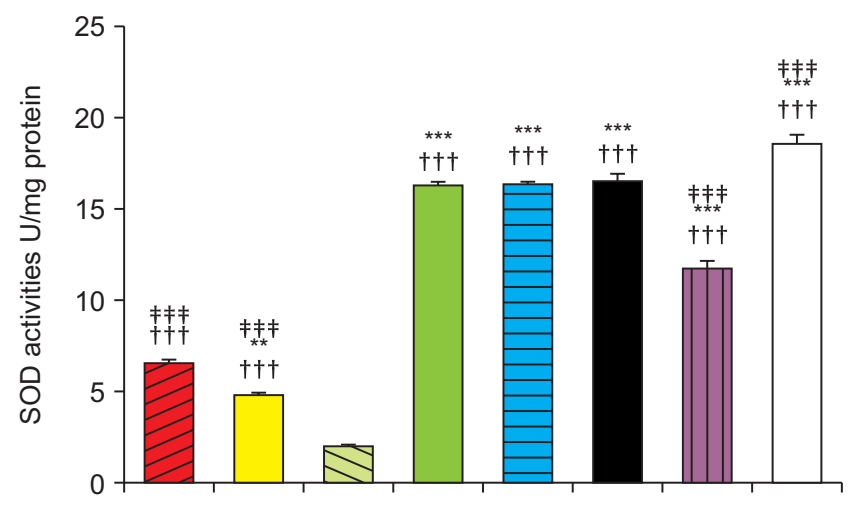

$\square$ Normal control
Sham control
Ligated control
$30 \mathrm{mg} / \mathrm{kg}$ gabapentin
$30 \mathrm{mg} / \mathrm{kg}$ bromelain
$50 \mathrm{mg} / \mathrm{kg}$ bromelain
Pretreated $30 \mathrm{mg} / \mathrm{kg}$ bromelain
$\square \quad$ Pretreated $50 \mathrm{mg} / \mathrm{kg}$ bromelain

Fig. 4. Bromelain increases antioxidant enzymes and subdue lipid peroxidation and nitric oxide. (A) Reduced glutathione (GSH) concentration. (B) Superoxide dismutase (SOD) activities. (C) Malondialdehyde (MDA) concentration. $* * P<0.01$, $* * * P<0.001$ compared with unligated control (normal control); ${ }^{\dagger \dagger \dagger} P<0.001$ compared with ligated control; and ${ }^{\ddagger \neq \ddagger} P<0.001$ compared with gabapentin (reference control).

A

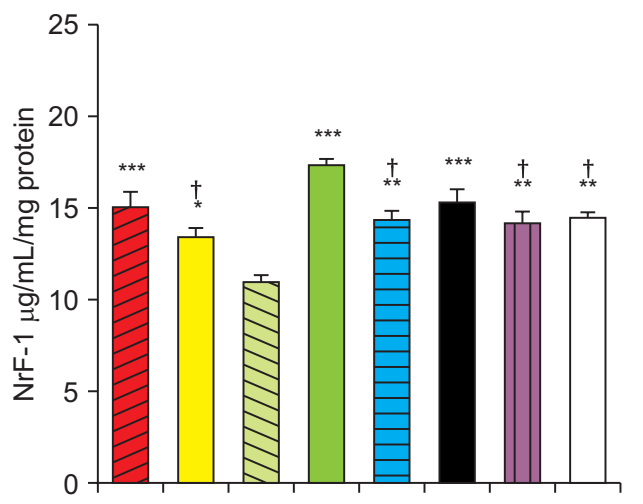

B

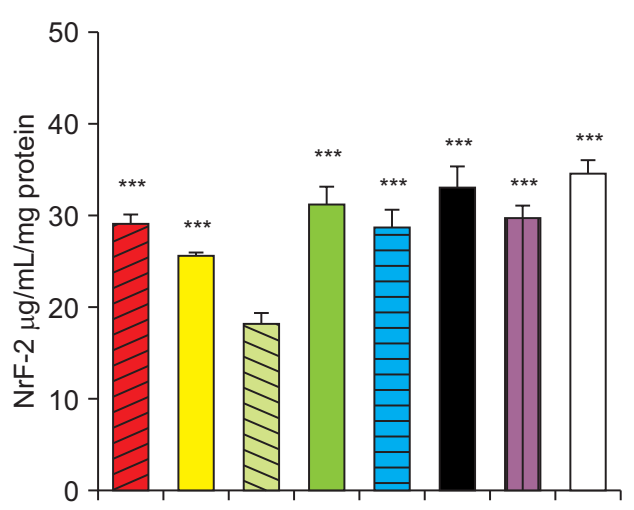

Normal control Sham control $\triangle$ Ligated control $30 \mathrm{mg} / \mathrm{kg}$ gabapentin $\Xi 30 \mathrm{mg} / \mathrm{kg}$ bromelain - $50 \mathrm{mg} / \mathrm{kg}$ bromelain

ㅁ. Pretreated $30 \mathrm{mg} / \mathrm{kg}$ bromelain Pretreated $50 \mathrm{mg} / \mathrm{kg}$ bromelain

Fig. 5. Effect of bromelain on transcription factors. (A) Nuclear factor erythroid-derived-2 related factor-1 (NrF-1). (B) NrF-2. $* P<0.05, * * P<0.01, * * * P$ $<0.001$ compared with ligated control; ${ }^{\dagger} P<0.05$ compared with gabapentin (reference control).

toms of neuropathic pain that can be modeled in animal studies [23]. The use of a hotplate for the screening of antinociceptive substances has been widely adopted and it has been linked to biochemical substances that act centrally at both the spinal and supra-spinal levels [24,25].

This study suggested that bromelain is effective in reversing the thermal hyperalgesic symptoms of neuropathy. Interestingly, pretreatment with bromelain prevented sciatic nerve ligated Wistar rats from developing thermal hyperalgelsia. This indicates that pretreatment with bromelain mitigated neuronal plasticity that could have resulted in thermal hyperalgesia. Findings from this study suggest that bromelain possess a similar antihyperalgesic effect compared to gabapentin, an anticonvulsant drug.

A mechanical allodynic symptom to tactile stimulus was significantly improved by bromelain in sciatic nerve 

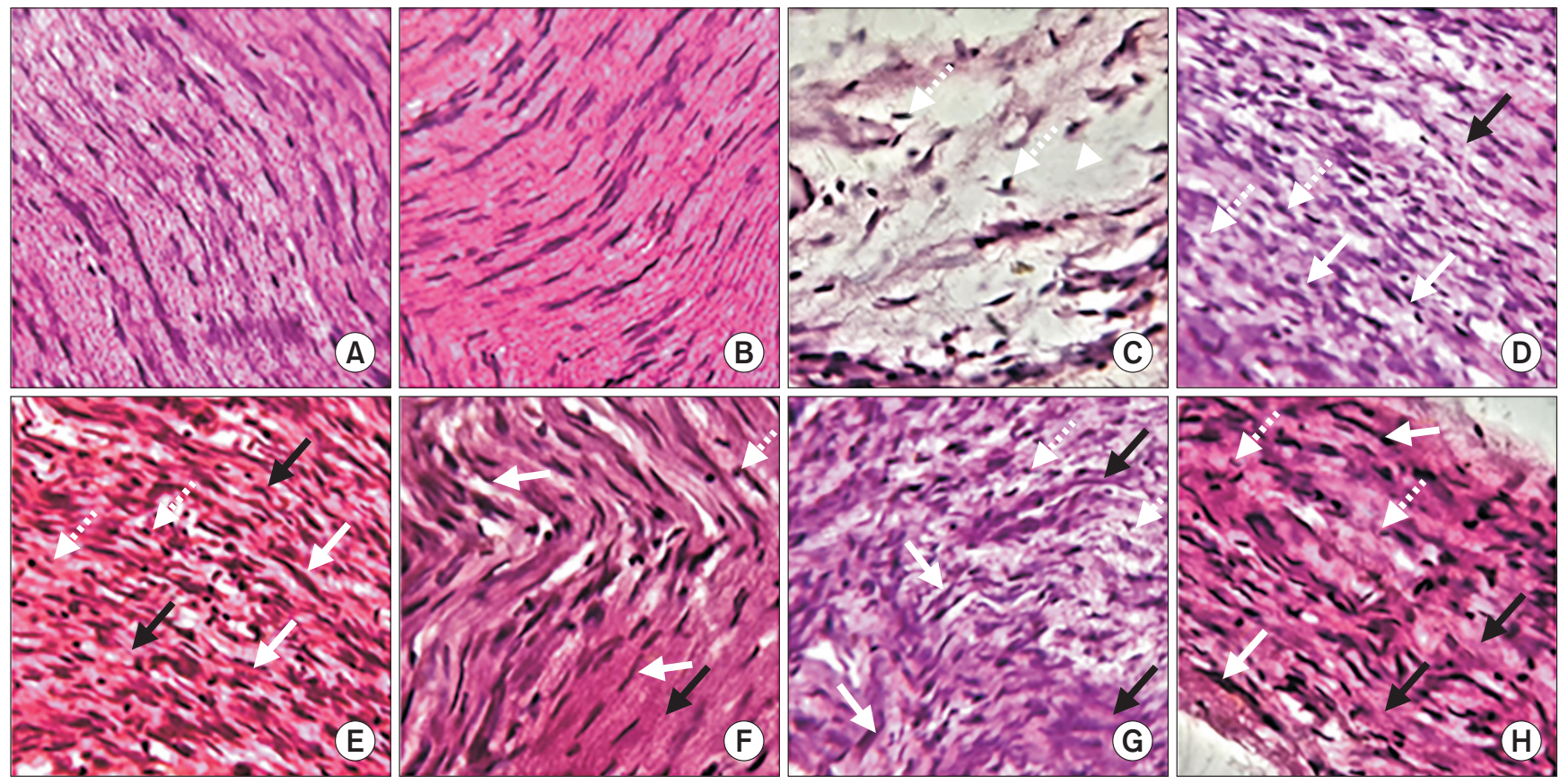

Fig. 6. Bromelain improved myelinated (white arrows) and unmyelinated nerve (black arrows) integrity. It attenuated swollen and degeneration of myelinated neurons (white arrows) and increases occurrence of Schwan's cells. Arrowhead indicates swollen and deranged non-myelinated neurons while dashed arrows indicate reduced and swollen myelinated neurons (hematoxylin and eosin stain, $\times 400)$. (A) Normal control, (B) sham control, (C) ligated control, (D) reference control (30 mg/kg gabapentin), (E) low dose bromelain (30 mg/kg), (F) high dose bromelain (50 mg/kg), (G) pre-treated low dose bromelain (30 mg/kg), (H) pre-treated high dose bromelain.

ligated rats. Meanwhile, mechanical allodynic symptoms developed in bromelain pretreated sciatic nerve ligated rats, but were subsequently mitigated as the treatment progressed. The results imply that pretreatment of rats with bromelain before the ligation of their sciatic nerves is more effective at mitigating the development of thermally induced allodynia than mechanically induced allodynia. This is indicative that bromelain effectively stabilized the A-delta and C-fibers conveying temperature relaying pain. On the other hand, bromelain could not influence the development of $\beta$ fiber pathology which elicits tactile allodynia. Also, nociceptive pain and thermal allodynia share the same fiber tracts (A $\delta$ and C fiber), while this is not the case with mechanical allodynia.

The need to evaluate the sciatic function is important because it reveals the extent of nerve demyelination and its dysfunctions. Bromelain improved the sciatic nerve function in the ligated animals. Increased sciatic function has been linked with improvement in peripheral nerve regeneration and correlated with indices of muscular strength, as well as the electrophysiology and morphology of the peripheral nerves [26].

This study shows that bromelain improved sciatic nerve structural integrity. The improved sciatic nerve structural architecture in the bromelain groups may be responsible for the improved sciatic function index obtained in these groups. A study by Monte-Raso et al. [27] showed that mor- phological recovery of the sciatic nerve correlates with its functional recovery. Pretreating rats with bromelain before they were rendered neuropathic did not significantly alter the derangement in the functional index when the pretreated groups were compared with the groups administered bromelain post-ligation. This indicates that bromelain could be used primarily as a therapeutic agent rather than as a prophylaxis. This is probably due to the inability of bromelain to prevent the development of the mechanical allodynia component as discussed earlier, and since the well-being of the animals would depend entirely on the complete absence of these indices of neuropathic pain, this explains why the functional index shows that the animals pretreated with bromelain followed the same trend as those not pre-treated.

The neuroprotective activities of bromelain were investigated via an assessment of its effects on antioxidant enzymes, LPO levels, and nuclear transcription factors. This study has shown that CCI induces inactivation of nuclear transcription factors (NrF-1 and NrF-2). Reduced activities of the nuclear transcription factors compromised the synthesis of antioxidant enzymes and its downstream signal molecules. This may explain the reason why SOD activities and GSH level were reduced in the ligated control group. It is hereby suggested that the reduction in the antioxidant defense system in the CCI model of neuropathic pain is associated with decreased activities in nuclear factor regula- 
tory protein and neuroprotective efficiency.

Structural disruption of the sciatic nerve has been linked with accumulation of ROS in the nervous system [4]. ROS increases phosphorylation of both peripheral and central sensory receptors that mediate hypersensitivities [5]. This study showed that bromelain promotes the activation of NrF-1 and NrF-2 and subsequently stimulates their translocation. It is therefore presumed that increases in antioxidant enzymes, such as SOD and GSH activities was due to the stimulating effect of bromelain on transcription factors. Studies have reported that NrF-1 and NrF-2 bind at the antioxidant response element of the DNA that stimulate the expression of genes which regulate enzymes involved in the synthesis of antioxidant enzymes [8].

Reduction in the level of MDA observed in this study is another evidence that shows the neuroprotective effect of bromelain. This is indicative of reduced neuronal LPO and ROS. Reduced ROS effects from bromelain may account for its antinociceptive activities. It has been suggested that ROS trigger the activation of sensory transient receptors (vanilloids) and mediate the released of chemical mediators that result in hyperalgesia $[28,29]$.

Gabapentin was used as a reference drug for this study. Gabapentin binds with the $\alpha 2 \delta$ subunit of the calcium channel [30]. Findings from this study revealed that gabapentin reverses both thermal and mechanical hypersensitivities, a finding which is in consonant with those of Kim et al. [31] in an animal model of neuropathic pain. The antinociceptive effects of gabapentin in a CCI model of neuropathic pain were similar to those of bromelain, although pretreatment with bromelain has a stronger effect on thermal hyperalgesic symptoms and the SFI.

In conclusion, this study demonstrated that bromelain possesses strong antinociceptive effects by reducing the symptoms associated with neuropathic pain. This antinociceptive effect of bromelain was mediated by increased activities of NrF-1 and NrF-2 which could stimulate the synthesis of antioxidant enzymes. The inactivation of these regulatory factors (NrF-1 and NrF-2) is crucial for the development of neuropathic pain. It is hereby proposed that increased activities of NrF-1 and NrF-2 by bromelain increased the SOD and GSH activities in the nerve and glial cells. This ultimately enhanced the antinociceptive properties of bromelain. Therefore, bromelain maybe a prospective therapy to be considered for the treatment of neuropathic pain.

\section{ACKNOWLEDGMENTS}

Our special appreciation goes to Mr. Sodiq Ishola and Mr. Bakare Habeeb for their immense technical support dur- ing the course of this study. We would also like to express appreciation to Dr. Lukuman Oyewole for donating the phosphate buffer used in this study.

\section{CONFLICT OF INTEREST}

No potential conflict of interest relevant to this article was reported.

\section{FUNDING}

No funding to declare.

\section{ORCID}

Ahmed Olalekan Bakare, https://orcid.org/0000-0003-3936-1228

Bamidele Victor Owoyele, https://orcid.org/0000-0003-3503-9338

\section{REFERENCES}

1. Jensen TS, Finnerup NB. Allodynia and hyperalgesia in neuropathic pain: clinical manifestations and mechanisms. Lancet Neurol 2014; 13: 924-35.

2. Muthuraman A, Singh N, Jaggi AS, Ramesh M. Drug therapy of neuropathic pain: current developments and future perspectives. Curr Drug Targets 2014; 15: 210-53.

3. Kanyadhara S, Dodoala S, Sampathi S, Punuru P, Chinta G. Ethanolic extract of Aloe vera ameliorates sciatic nerve ligation induced neuropathic pain. Anc Sci Life 2014; 33: 208-15.

4. Duggett NA, Griffiths LA, McKenna OE, de Santis V, Yongsanguanchai N, Mokori EB, et al. Oxidative stress in the development, maintenance and resolution of paclitaxel-induced painful neuropathy. Neuroscience 2016; 333: 13-26.

5. Zhang EJ, Song CH, Ko YK, Lee WH. Intrathecal administration of mesenchymal stem cells reduces the reactive oxygen species and pain behavior in neuropathic rats. Korean J Pain 2014; 27: 239-45.

6. Kim HK, Zhang YP, Gwak YS, Abdi S. Phenyl N-tert-butylnitrone, a free radical scavenger, reduces mechanical allodynia in chemotherapy-induced neuropathic pain in rats. Anesthesiology 2010; 112: 432-9.

7. Negi G, Kumar A, Joshi RP, Sharma SS. Oxidative stress and Nrf2 in the pathophysiology of diabetic neuropathy: old perspective with a new angle. Biochem Biophys Res Commun 2011; 408: 1-5.

8. Biswas M, Chan JY. Role of Nrf1 in antioxidant response element-mediated gene expression and beyond. Toxicol Appl Pharmacol 2010; 244: 16-20. 
9. Li W, Kong AN. Molecular mechanisms of Nrf2-mediated antioxidant response. Mol Carcinog 2009; 48: 91-104.

10. Majid OW, Al-Mashhadani BA. Perioperative bromelain reduces pain and swelling and improves quality of life measures after mandibular third molar surgery: a randomized, double-blind, placebo-controlled clinical trial. J Oral Maxillofac Surg 2014; 72: 1043-8.

11. Golezar S. Ananas comosus Effect on perineal pain and wound healing after episiotomy: a randomized double-blind placebo-controlled clinical trial. Iran Red Crescent Med J 2016; 18: e21019.

12. Sudjarwo SA. Anti-inflammatory and analgesic effect of bromelain in mice and rats. Univ Med 2005; 24: 155-60.

13. Jiang X, Yan Q, Liu F, Jing C, Ding L, Zhang L, et al. Chronic trans-astaxanthin treatment exerts antihyperalgesic effect and corrects co-morbid depressive like behaviors in mice with chronic pain. Neurosci Lett 2018; 662: 36-43.

14. Kilic FS, Sirmagul B, Yildirim E, Oner S, Erol K. Antinociceptive effects of gabapentin \& its mechanism of action in experimental animal studies. Indian J Med Res 2012; 135: 630 5.

15. Zhu YF, Wu Q, Henry JL. Changes in functional properties of A-type but not C-type sensory neurons in vivo in a rat model of peripheral neuropathy. J Pain Res 2012; 5: 175-92.

16. Zhang MT, Wang B, Jia YN, Liu N, Ma PS, Gong SS, et al. Neuroprotective effect of liquiritin against neuropathic pain induced by chronic constriction injury of the sciatic nerve in mice. Biomed Pharmacother 2017; 95: 186-98.

17. Bain JR, Mackinnon SE, Hunter DA. Functional evaluation of complete sciatic, peroneal, and posterior tibial nerve lesions in the rat. Plast Reconstr Surg 1989; 83: 129-38.

18. Hunter FE Jr, Gebicki JM, Hoffsten PE, Weinstein J, Scott A. Swelling and lysis of rat liver mitochondria induced by ferrous ions. J Biol Chem 1963; 238: 828-35.

19. Gutteridge JM, Wilkins S. Copper-dependent hydroxyl radical damage to ascorbic acid: formation of a thiobarbituric acid-reactive product. FEBS Lett 1982; 137: 327-30.

20. Misra HP, Fridovich I. The role of superoxide anion in the autoxidation of epinephrine and a simple assay for superoxide dismutase. J Biol Chem 1972; 247: 3170-5.
21. Ellman GL. Tissue sulfhydryl groups. Arch Biochem Biophys 1959; 82: 70-7.

22. Muthuraman A, Diwan V, Jaggi AS, Singh N, Singh D. Ameliorative effects of Ocimum sanctum in sciatic nerve transection-induced neuropathy in rats. J Ethnopharmacol 2008; 120: $56-62$

23. Moini Zanjani T, Ameli H, Labibi F, Sedaghat K, Sabetkasaei M. The attenuation of pain behavior and serum COX-2 concentration by curcumin in a rat model of neuropathic pain. Korean J Pain 2014; 27: 246-52.

24. Ishola IO, Agbaje EO, Adeyemi OO, Shukla R. Analgesic and anti-inflammatory effects of the methanol root extracts of some selected Nigerian medicinal plants. Pharm Biol 2014; 52: 1208-16.

25. Owoyele BV, Bakare AO. Analgesic properties of aqueous bark extract of Adansonia digitata in Wistar rats. Biomed Pharmacother 2018; 97: 209-12.

26. Shen N, Zhu J. Application of sciatic functional index in nerve functional assessment. Microsurgery 1995; 16: 552-5.

27. Monte-Raso VV, Barbieri CH, Mazzer N, Yamasita AC, Barbieri G. Is the Sciatic Functional Index always reliable and reproducible? J Neurosci Methods 2008; 170: 255-61.

28. Hassler SN, Johnson KM, Hulsebosch CE. Reactive oxygen species and lipid peroxidation inhibitors reduce mechanical sensitivity in a chronic neuropathic pain model of spinal cord injury in rats. J Neurochem 2014; 131: 413-7.

29. Goel R, Tyagi N. Potential contribution of antioxidant mechanism in the defensive effect of lycopene against partial sciatic nerve ligation induced behavioral, biochemical and histopathological modification in wistar rats. Drug Res (Stuttg) 2016; 66: 633-8.

30. Luo ZD, Chaplan SR, Higuera ES, Sorkin LS, Stauderman KA, Williams ME, et al. Upregulation of dorsal root ganglion (alpha)2(delta) calcium channel subunit and its correlation with allodynia in spinal nerve-injured rats. J Neurosci 2001; 21: 1868-75.

31. Kim MJ, Lee JH, Jang JU, Quan FS, Kim SK, Kim W. The efficacy of combination treatment of gabapentin and electroacupuncture on paclitaxel-induced neuropathic pain. Korean J Physiol Pharmacol 2017; 21: 657-66. 